\title{
A THEOREM ABOUT FRACTIONAL INTEGRALS
}

\section{NICOLAAS DU PLESSIS}

It is a classical result that if $f(x)$ is Lebesgue integrable in a finite interval, then it is finite p.p. One is led to enquire about the behaviour of the fractional integral $f_{\beta}$ of $f$.

Suppose, for convenience, that $f(x)$ is defined in $[0,2 \pi]$. We then have the following theorem.

THEOREM. If $f \in L^{q}[0,2 \pi]$, then:

(a) For $0<\alpha<1,2<q<\infty, f_{\alpha / q}$ is finite everywhere except in a set which is of zero $\beta$-capacity for every $\beta>1-\alpha$.

(b) For $0<\alpha<1,1 \leqq q \leqq 2, f_{\alpha / q}$ is finite everywhere except possibly in a set of zero $(1-\alpha)$-capacity.

Both (a) and (b) are best possible.

Since, as is well known, the Riemann-Liouville and the Weyl versions of the fractional integral of a function in $L^{q}$ differ by a bounded function, this theorem holds for both versions if it is shown to hold for either one. Use is made of this fact in what follows.

1. In this section I prove a lemma which is possibly of greater interest than the theorem itself.

Lemma. Let $\mu(x)$ be a nondecreasing bounded function in $[0,2 \pi]$. Let, for $0<\alpha<1$,

$$
V_{1-\alpha}=\sup \int_{0}^{2 \pi}|x-t|^{\alpha-1} d \mu(t) \quad \text { for } x \in[0,2 \pi] .
$$

Then, for every $\epsilon>0$ and $1<q<2$,

$$
M_{q-\epsilon}\left[\int_{0}^{2 \pi}|x-t|^{\alpha / q^{\prime}-1} d \mu(t)\right] \leqq A(\alpha, \epsilon) V_{1-\alpha}^{1 /(q-\epsilon)^{\prime}},
$$

where $A(\alpha, \epsilon)$ is a constant depending only on $\alpha$ and $\epsilon$, and, for $2 \leqq q \leqq \infty$,

$$
M_{q}\left[\int_{0}^{2 \pi}|x-t|^{\alpha / q^{\prime}-1} d \mu(t)\right] \leqq A(\alpha) V_{1-\alpha}^{1 / q^{\prime}},
$$

where $A(\alpha)$ is a constant depending only on $\alpha$.

We have

Received by the editors February 26, 1952. 


$$
\int_{0}^{2 \pi}|x-t|^{\alpha / q^{\prime}-1} d \mu(t)=\int_{0}^{2 \pi}|x-t|^{-\alpha / q} d v_{x}(t)
$$

where

$$
v_{x}(t)=\int_{0}^{t}|x-s|^{\alpha-1} d \mu(s)
$$

Consequently, using Hölder's inequality,

$$
\begin{aligned}
& \left\{\int_{0}^{2 \pi}|x-t|^{\alpha / q^{\prime}-1} d \mu(t)\right\}^{q-\epsilon} \\
& \qquad\left\{\int_{0}^{2 \pi}|x-t|-\alpha(q-\epsilon) / q d v_{x}(t)\right\}\left\{\int_{0}^{2 \pi} d v_{x}(t)\right\}^{(q-\epsilon) /(q-\epsilon) \prime}
\end{aligned}
$$

and this does not exceed

$$
\left\{\int_{0}^{2 \pi}|x-t|^{\alpha \epsilon / q-1} d \mu(t)\right\} V_{1-\alpha}^{(q-\epsilon) /(q-\epsilon)^{\prime}} .
$$

Thus, the left-hand side of (1) does not exceed

$$
V^{1 /(q-\epsilon)^{\prime}}\left\{\int_{0}^{2 \pi} d \mu(t) \int_{0}^{2 \pi}|x-t|^{\alpha \epsilon / q-1} d x\right\}^{1 /(q-\epsilon)},
$$

which gives (1). It is surprising that so crude an argument gives a best possible result.

To prove (2) I first show the result true for $q=2$ and then that this implies its truth for $q>2$. For this latter portion of the proof $I$ am indebted to Professor J. E. Littlewood.

We have first, inverting the order of integration,

$$
\begin{aligned}
& M_{2}^{2}\left[\int_{0}^{2 \pi}|x-t|^{\alpha / 2-1} d \mu(t)\right] \\
& \quad=\int_{0}^{2 \pi} \int_{0}^{2 \pi} \int_{0}^{2 \pi}|x-t|^{\alpha / 2-1}|x-s|^{\alpha / 2-1} d x d \mu(t) d \mu(s)
\end{aligned}
$$

In the inner integral we make the substitution $x-t=(s-t) u$ and find that the integral does not exceed

$$
|s-t|^{\alpha-1} \int_{-\infty}^{\infty}|u(1-u)|^{\alpha / 2-1} d u=B(\alpha)|s-t|^{\alpha-1} .
$$

Consequently, the right-hand side of (3) is dominated by 


$$
B(\alpha) \int_{0}^{2 \pi} \int_{0}^{2 \pi}|s-t|^{\alpha-1} d \mu(t) d \mu(s) \leqq B(\alpha) V_{1-\alpha}(\mu(2 \pi)-\mu(0)),
$$

which gives the result for $q=2$.

For $q>2$, we have

$$
\begin{aligned}
M_{q}^{q}\left[\int_{0}^{2 \pi}|x-t|^{\alpha / q^{\prime}-1} d \mu(t)\right] \\
\quad=\int_{0}^{2 \pi} d x\left\{\int_{0}^{2 \pi}|x-t|^{((q-2) / q)(\alpha-1)}|x-t|^{(\alpha-2) / q} d \mu(t)\right\}^{q}
\end{aligned}
$$

and this, by Hölder's inequality, does not exceed

$$
\int_{0}^{2 \pi} d x\left\{\int_{0}^{2 \pi}|x-t|^{\alpha-1} d \mu(t)\right\}^{q-2}\left\{\int_{0}^{2 \pi}|x-t|^{\alpha / 2-1} d \mu(t)\right\}^{2},
$$

which is, in turn, dominated by

$$
V_{1-\alpha}^{q-2} M_{2}^{2}\left[\int_{0}^{2 \pi}|x-t|^{\alpha / 2-1} d \mu(t)\right] \leqq A(\alpha) V_{1-\alpha,}^{q-1}
$$

which gives the result for $q>2$.

2. Proof of the theorem. Suppose that $E$ is a subset of $[0,2 \pi]$. If a nondecreasing $\mu(x)$ is such that

$$
\int_{E} d \mu(t)=\int_{0}^{2 \pi} d \mu(t)=1,
$$

we say that $\mu(x)$ is a distribution concentrated on $E$. If, further, for any $\beta(0<\beta<1)$ there is a $\mu(x)$ concentrated on $E$ such that

$$
V_{\beta}=\sup \int_{0}^{2 \pi}|x-t|^{-\beta} d \mu(t) \quad \text { for all } x \in[0,2 \pi]
$$

is finite, then $E$ is said to be of positive $\beta$-capacity. Otherwise $E$ is said to be of zero $\beta$-capacity. This definition is equivalent to that given by Salem and Zygmund [1].

Clearly, if $E$ is of positive $\beta$-capacity, it is of positive $\gamma$-capacity for all $\gamma<\beta$. If it is of zero $\beta$-capacity, it is of zero $\gamma$-capacity for all $\gamma>\beta$.

We may, without loss of generality, assume $\int_{0}^{2 \pi} f(x) d x=0$ and use the Weyl fractional integral.

Let $f(x)$ have the Fourier series 


$$
\sum_{n=-\infty}^{\infty} c_{n} e^{i n x}
$$

where' signifies that $c_{0}=0$. Then

$$
f_{\alpha / q}(x)=\sum_{k=-\infty}^{\infty}(i k)^{-\alpha / q} c_{k} e^{i k x}
$$

and it is sufficient to show that $S_{n}$ is bounded outside a set of zero $\beta$-capacity, where $S_{n}=\sum_{-n}^{n}(i k)^{-\alpha / q} c_{k} e^{i k x}$ and $\beta=1-\alpha$ for $1 \leqq q \leqq 2$ and $\beta>1-\alpha$ for $q>2$.

Assume then that $S_{n}$ is unbounded in a set $E$ of positive $\beta$-capacity. Then, first, there is a distribution $\mu(x)$ concentrated on $E$ such that

$$
\int_{0}^{2 \pi}|x-t|^{-\beta} d \mu(t)
$$

is bounded for all $x$. Secondly, by a well known argument there is a function $n(x) \leqq n$, taking only integer values, such that

$$
\int_{0}^{2 \pi} S_{n(x)}(x) d \mu(x)
$$

exists and is unbounded as $n \rightarrow \infty$. I show this last to be impossible. For

$$
\begin{aligned}
\int_{0}^{2 \pi} S_{n(x)}(x) d \mu(x) & =\int_{0}^{2 \pi} \sum_{k=-n(x)}^{n(x)}(i k)^{-\alpha / q} c_{k} e^{i k x} d \mu(x) \\
= & \int_{0}^{2 \pi} \int_{0}^{2 \pi} f(t) \sum_{k=-n(x)}^{n(x)}(i k)^{-\alpha / q} e^{i n(x-t)} d t d \mu(x) .
\end{aligned}
$$

Now

$$
\left|\sum_{k=-n(x)}^{n(x)}(i k)^{-\alpha / q} e^{i k(x-t)}\right| \leqq C|x-t|^{\alpha / q-1}
$$

so

$$
\begin{aligned}
\left|\int_{0}^{2 \pi} S_{n(x)}(x) d \mu(x)\right| & \leqq C \int_{0}^{2 \pi}|f(t)|\left\{\int_{0}^{2 \pi}|x-t|^{\alpha / q-1} d \mu(x)\right\} d t \\
& \leqq C M_{q}(f) M_{q^{\prime}}\left[\int_{0}^{2 \pi}|x-t|^{\alpha / q-1} d \mu(x)\right] .
\end{aligned}
$$

Now $M_{q}(f)<\infty$ by hypothesis and we have only to show 


$$
M_{q^{\prime}}\left[\int_{0}^{2 \pi}|x-t|^{\alpha / q-1} d \mu(x)\right]
$$

bounded.

If $1 \leqq q \leqq 2$, then $q^{\prime} \geqq 2$ and (2) of $\$ 1$ immediately shows this.

If $q>2$, we write $\beta=1-\gamma$. Since $\gamma<\alpha$, there is an $r<q$ such that $\alpha / q=\gamma / r$. We may suppose $\beta$ so near to $1-\alpha$ that $2<r<q$, since if we show the result for all such $\beta$ it will immediately follow for all larger $\beta$. We may now rewrite (1) in the form

$$
M_{r^{\prime}-\epsilon}\left[\int_{0}^{2 \pi}|x-t| \gamma / r-1 d \mu(x)\right],
$$

which, since $r^{\prime}<2$, is shown to be bounded by invoking (1) of $\S 1$.

This gives the result.

3. The theorem is best possible. Construct the set $S$ as follows. Let $\left\{\xi_{n}\right\}$ be any sequence such that $0<\xi_{n}<1 / 2$. From $S_{0}=[0,2 \pi]$ remove the open concentric interval of length $2 \pi\left(1-2 \xi_{1}\right)$, thus leaving the set $S_{1}$. From each of the intervals in $S_{1}$, of length $2 \pi \xi_{1}$, remove an open concentric interval of length $2 \pi \xi_{1}\left(1-2 \xi_{2}\right)$, leaving a set $S_{2}$ consisting of four closed intervals each of length $2 \pi \xi_{1} \xi_{2}$. Continuing in this way, we are left, after the $k$ th removal, with a set $S_{k}$ consisting of $2^{k}$ closed intervals each of length $2 \pi \xi_{1} \xi_{2} \cdots \xi_{k}$. Consequently $m S_{k}=2 \pi 2^{k} \xi_{1} \xi_{2} \cdots \xi_{k}$.

It is known $\left[1\right.$, p. 40] that $S=\lim S_{k}$ will be of positive $\beta$-capacity if and only if

$$
\sum_{k=1}^{\infty} 2^{-k}\left(\xi_{1} \xi_{2} \cdots \xi_{k}\right)^{-\beta}<\infty .
$$

Define $\left\{f_{n}(x)\right\}$ on $[0,2 \pi]$ by

$$
\begin{aligned}
f_{0}(x) & =0 \text { in } S_{0}, \\
f_{n}(x) & =\left(\xi_{1} \xi_{2} \cdots \xi_{n}\right)^{-\alpha / q_{n}-1} \text { in } S_{n} \\
& =f_{n-1}(x) \text { in } S_{0}-S_{n}
\end{aligned}
$$

for $n=1,2,3 \cdots$. Since $\left\{f_{n}(x)\right\}$ is an increasing sequence of measurable functions,

$$
f(x)=\lim _{n \rightarrow \infty} f_{n}(x)
$$

exists and is measurable.

It is easily seen that 
1952]

$$
f(x)=\left(\xi_{1} \xi_{2} \cdots \xi_{n}\right)^{-\alpha / q_{n}-1} \text { on } S_{n}-S_{n+1}, \quad n=1,2, \cdots,
$$

so that

$$
\begin{aligned}
\int_{0}^{2 \pi}|f(x)|^{q} d x & =\sum_{n=1}^{\infty} \int_{S_{n}-S_{n+1}}|f(x)|^{q} d x \\
& =\sum_{n=1}^{\infty}\left(\xi_{1} \xi_{2} \cdots \xi_{n}\right)^{-\alpha} n^{-q}\left[m S_{n}-m S_{n+1}\right] \\
& =\sum_{n=1}^{\infty}\left(1-2 \xi_{n+1}\right) 2^{n}\left(\xi_{1} \xi_{2} \cdots \xi_{n}\right)^{1-\alpha} n^{-q}
\end{aligned}
$$

For $q>2$ we may choose $\delta>0$ so that $2(1+\delta)<q$ and then put

$$
2 \xi_{n}^{1-\alpha}=1+(1+\delta) n^{-1}
$$

Then

$$
2^{-k}\left(\xi_{1} \xi_{2} \cdots \xi_{k}\right)^{\alpha-1}=O\left(k^{-1-\delta}\right)
$$

and so (1) with $\beta=1-\alpha$ is satisfied, showing $S$ to be of positive $(1-\alpha)$ capacity.

Further (2) is clearly finite so that $f(x) \in L^{q}$.

Let $x$ be any point of $S$. Now $S_{k}-S_{k+1}$ consists of $2^{k}$ intervals each of length $2 \pi \xi_{1} \xi_{2} \cdots \xi_{k}\left(1-2 \xi_{k+1}\right)$ none of which contain $x$. However, one of these intervals $I_{k}$ is contained in an interval of $S_{k}$ which contains $x$. Consequently

$$
\int_{0}^{2 \pi}|x-t|^{\alpha / q-1} f(t) d t=\sum_{k=1}^{\infty} \int_{S_{k}-S_{k+1}} \geqq \sum_{k=1}^{\infty} \int_{I_{k}}
$$

and this last is itself greater than

$$
\begin{aligned}
(2 \pi)^{\alpha / q} \cdot \sum_{k=1}^{\infty}\left(\xi_{1} \xi_{2} \cdots \xi_{k}\right)^{\alpha / q-1}\left(\xi_{1} \xi_{2} \cdots \xi_{k}\right)^{-\alpha / q} k^{-1}\left(\xi_{1} \cdots \xi_{k}\right)\left(1-2 \xi_{k+1}\right) \\
=(2 \pi)^{\alpha / q} \sum_{k=1}^{\infty}\left(1-2 \xi_{k+1}\right) k^{-1}=+\infty
\end{aligned}
$$

and so $\int_{0}^{2 \pi}|x-t|^{\alpha / q-1} f(t) d t=+\infty$ at every point of $S$.

Now $f(x)=f(2 \pi-x)$ so that

$$
\int_{0}^{2 \pi}|x-t|^{\alpha / q-1} f(t) d t=f_{\alpha / q}(x)+f_{\alpha / q}(2 \pi-x)
$$

where $f_{\alpha / g}$ now denotes the Riemann-Liouville fractional integral.

It follows that $f_{\alpha / q}(x)$ must be infinite in a set of positive $(1-\alpha)$ - 
capacity. For, if not, suppose $f_{\alpha / g}(x)$ infinite in a set $M$ of zero $(1-\alpha)$-capacity. Then $f_{\alpha / q}(2 \pi-x)$ is infinite in the mirror-image $\bar{M}$ of $M$ about $x=\pi$. Also $M+\bar{M}=S$, and since both $M$ and $\bar{M}$ are of zero $(1-\alpha)$-capacity, so is $S$. This contradiction gives the required result and shows part (b) of the theorem to be best possible.

Next, let $\beta$ be any positive number less than $1-\alpha$ and let $\xi$ be such that

$$
2 \xi^{(1-\alpha+\beta) / 2}=1 .
$$

Now consider the set $S$ with $\xi_{n}=\xi$ for all $n$. Since $2 \xi^{\beta}>1, S$ is of positive $\beta$-capacity. Defining $f(x)$ as before we use exactly the same argument to show $f_{\alpha / q}(x)=+\infty$ in a set of positive $\beta$-capacity. Furthermore, since $2 \xi^{1-\alpha}<1$, (2) is bounded, showing $f \in L^{q}$. This proves part (a) of the theorem to be best possible.

In passing, we may note that it has here been shown that a function in $L^{q}$ for any $q$ may be infinite in a set which is "only just" of measure zero. More precisely, given any $\beta<1$ and any $q>1$, there is a function in $L^{q}$ which is infinite in a set of positive $\beta$-capacity, i.e., in a set of positive $\beta$-Hausdorff measure.

4. The lemma of $\S 1$ is best possible. Consider e.g., (2) of $\S 1$. Suppose this is not best possible, i.e., that there is an $\epsilon>0$ for which, in general,

$$
M_{q+\epsilon}\left[\int_{0}^{2 \pi}|x-t|^{\alpha / q^{\prime}-1} d \mu(t)\right]<\infty .
$$

If, then, $f(x) \in L^{(q+\epsilon) '}$, we may say

$$
\left|\int_{0}^{2 \pi} S_{n(x)}(x) d \mu(x)\right| \leqq C M_{(q+\epsilon)^{\prime}}(f) M_{q+\epsilon}\left[\int_{0}^{2 \pi}|x-t|^{\alpha / q^{\prime}-1} d \mu(t)\right],
$$

which is bounded. This would imply that (b) of the theorem is not best possible. Since it is, we have shown (2) best possible. A similar argument using (a) would show (1) best possible.

\section{REFERENCES}

1. R. Salem and A. Zygmund, Capacity of sets and Fourier series, Trans. Amer. Math. Soc. vol. 59 (1946) pp. 23-41.

2. O. Frostman, Potential d'équilibre et capacité des ensembles, Meddelande från Lunds Univ. Mat. Sem. vol. 3 (1935).

RHODES UNIVERSITY 